\title{
Du guide SAEL aux sites de français langue étrangère
}

\author{
Nadège Lechevrel \\ Université d'Angers et EHESS (LIAS-Institut Marcel Mauss) \\ nadege.lechevrel@ehess.fr
}

\section{Introduction}

En novembre 2009 est paru le guide SAEL du projet multilatéral Comenius SAEL (http://www.eurosael.eu/fr/projet/les-partenaires-19.html). Il s'agit d'un Guide pratique pour la conception, l'animation et l'amélioration de sites d'accompagnement pour les enseignants de langues, produit par le Centre international d'études pédagogiques (CIEP), l'Association nationale des enseignants de français (ANIF) et Europees Platform - internationaliseren in onderwijs. Dans le premier chapitre « Pourquoi créer un site d'accompagnement à destination des enseignants de langues?», le guide souligne d'une part que le site d'accompagnement est au service de la politique linguistique (en ce qu'il permet une meilleure diffusion des transformations de l'enseignement des langues comme l'introduction du CECRL ${ }^{1}$ ); d'autre part, qu'il constitue un moyen d'impulser une réflexion sur les matériaux, les outils et les méthodes mis en ouvre au niveau de l'institution. C'est dans ce cadre précis que nous avons souhaité aller de la lecture du guide SAEL, qui nous est adressé, à une réflexion sur les techniques utilisées dans la conception de sites de langue, institutionnels, sous tutelle étatique, ou indépendants. En effet, comme le souligne le guide dans son introduction, « depuis une dizaine d'années, l'enseignement des langues connait des mutations profondes, provoquées par l'introduction de nouvelles approches (...) et du développement des TICE » (Bonnet, 2009:6). La maîtrise des TICE est ainsi déterminante pour participer pleinement au développement de la formation en ligne (ou e-learning ${ }^{2}$ ) et pour le maintien de la diffusion et de l'enseignement-apprentissage du français; les sites d'accompagnement à destination des enseignants de langues sont donc importants car ils participent à une politique de sensibilisation générale à l'usage des nouvelles technologies. Dans ce domaine, certains sites utilisent des outils attractifs pour apprendre les langues que l'on pourrait voir se diffuser pour les sites d'enseignement de la langue française. Nous souhaitons montrer qu'ils sont riches d'enseignement et soulignons la façon dont ils peuvent guider nos pratiques ou constituer des modèles pour l'élaboration de sites internet FLE (à destination des enseignants et/ou des apprenants) qu'il s'agisse de portails, de services, de centres virtuels ou de serveurs de l'éducation.

Pour réaliser notre enquête, nous avons comparé des données provenant de sites ESL (English as a second language) et FLE (français langue étrangère). Ceci a permis de relever une différence significative dans le domaine des techniques utlisées, à partir desquelles nous soumettons nos observations espérant contribuer ainsi à l'amélioration de l'élaboration des sites à destination des apprenants, outils et techniques pouvant être délivrés sur les sites d'accompagnement pour les enseignants, décrits par le guide SAEL. De telles améliorations nous paraissent indispensables pour que les sites de FLE soient de véritables outils au service de la politique linguistique, c'est-à-dire conçus comme ayant vocation à soutenir une meilleure diffusion de la langue française et de ses variétés dans le monde.

\section{Enquête}

\subsection{Préambule}

Mirage pédagogique ou réelle transformation du paysage éducatif, le développement des TICE (technologies de l'information et de la communication pour l'éducation) a bien eu lieu. Toutes les universités se dotent ou se sont dotées, il y a déjà quelques années, d'environnements numériques de travail (dits ENT) et encouragent leur personnel enseignant à les utiliser pour accompagner leur 
enseignement. On y dépose des polycopiés, des exercices, des documents complémentaires, mais on s'en sert aussi comme espace de dialogue (par le biais des forums) et de débats, d'extension du cours et des $\mathrm{TD}$, et l'on peut même, si l'on y prend goût, en faire un espace attractif en y associant un souci graphique et ergonomique (choix des polices, des images, agencement des documents et fichiers de la page d'accueil, etc.). Les TICE ont par ailleurs « démultiplié les modalités d'accompagnement des enseignants et des apprenants et ouvert les stratégies de formation en rendant accessibles de nouveaux environnements et des espaces de communication » (Bonnet, $2009: 8$ ).

Dans le cadre des formations FLE, les étudiants sont de plus en plus amenés à réfléchir à la conception de sites internet pour accompagner leur enseignement de la langue française. C'est souvent à cette occasion qu'ils découvrent l'intérêt des TICE pour eux-mêmes et pour leurs futurs apprenants, tant du point de vue de l'enseignement-apprentissage des langues que celui plus engagé de la diffusion de la langue française. Cependant, les étudiants de cursus FLE semblent souvent s'interroger davantage sur la façon dont ils pourraient intégrer le volume de données trouvées en surfant sur la Toile au contenu de leurs cours, que sur la façon dont ils pourraient rendre ces sources accessibles aux apprenants en les intégrant pleinenemt à des activités pédagogiques (du type WebQuest). Cette observation montre à nouveau la nécessité de former les futurs enseignants non pas seulement à l'usage de l'internet, mais à la didactisation des ressources virtuelles ${ }^{3}$.

Ces cours de formation aux TICE leur pemettent également d'exprimer leurs impressions sur l'offre des sites internet. Les observations de nos étudiants ont conduit à faire plusieurs constats dont celui d'un manque d'harmonisation dans la palette des sites FLE offerts au public. La question des standards est de fait très souvent abordée, mais l'est essentiellement (et logiquement) du point de vue de la formation en ligne dispensée en cadre institutionnel (voir Educnet, « Normes et standards »). Par ailleurs, si les sites institutionnels sont les plus réussis, comme le remarquent la plupart des étudiants, ils ne sont pas pour autant les plus visités, ni les plus utilisés par les apprenants de français langue étrangère. Ces sites semblent d'ailleurs dérouter les futurs enseignants de FLE qui y voient de véritables vitrines pour la francophonie, mais trop peu d'outils accessibles aux apprenants débutants (de A1 à B2 du CECRL ${ }^{4}$ ), tant la langue utilisée à tous les niveaux (y compris pour la navigation à l'intérieur du site) ne peut véritablement être comprise que par les plus avancés ( $\mathrm{C} 1$ et $\mathrm{C} 2$ ). En termes d'utilité, leur usage est aussi réduit du fait qu'ils manquent de définir « l'architecture de l'information sur la base d'une hypothèse de parcours logique et cohérent de consultation des utilisateurs, or l'architecture de l'information ne doit pas être le simple reflet de la structure organisationnelle de l'administration émettrice $»^{5}$. Quant aux sites FLE indépendants, ce sont le plus souvent les acteurs eux-mêmes (formateurs ou étudiants) qui en sont les concepteurs autonomes et si ces sites parfois donnent l'impression d'un manque de professionnalisme, c'est certainement comme le note Monique Grandbastien (2004: 405) parce que « (...) les pédagogues conçoivent et décrivent de façon informelle des séquences d'activités pour des apprenants depuis des siècles et que pour la première fois dans l'histoire, on leur en demande une modélisation formelle interprétable par une machine, ce qui représente un saut conceptuel considérable. », d'où la nécessité, encore une fois, d'une formation aux TICE et de moyens financiers pour la conception de sites internet, sans qu'il ne soit question de parler de normes pour ces derniers. Aussi, mis à part les sites institutionnels à destination des formateurs ou d'un public francophone international, il reste aux apprenants débutants de très nombreux sites, pour la plupart non institutionnels, sur lesquels nous avons donc porté notre attention; ces sites, aux contenus de qualité, présentant néanmoins quelques caractéristiques jouant parfois en leur défaveur.

\subsection{Méthode}

Nous avons différencié les sites portails offrant du matériel pédagogique aux enseignants et étudiants en formation FLE (voir http://www.francparler.org/annuaire/fle.htm pour une liste détaillée), des sites institutionnels (pouvant recouper la première catégorie), des sites indépendants. Un rapide tour d'horizon par trois types de recherche internet : un référencement via l'outil Quarkbase avec le critère « site FLE » qui implique une identité numérique en ces termes (17 sites performants seulement); un référencement plus largement ciblé via CITO $^{6}$ (1204 ressources) et une simple recherche par moteur de recherche avec 
mots-clés ciblés également ${ }^{7}$. Nous avons ajouté à cela nos connaissances personnelles de sites internet de FLE et d'autres recensements comme celui qu'offre la page «Ressources FLE et langue française » (http://www.xtec.cat/ \%20sgirona/fle/links8lf.htm) du site «Les pages du FLE» de la Généralité de Catalogne (Departament d'Educació) ${ }^{8}$.

Nos corpus permettent d'identifier certains problèmes relevant en partie d'un manque de formation, d'où la nécessité et l'intérêt d'un Guide SAEL qui contient de nombreuses informations sur les aspects techniques. Nous nous concentrerons ici sur les points paraissant être les moins bien maîtrisés, mais étant les plus importants, et qui concernent principalement la maîtrise du référencement (ou identité numérique) et l'architecture de l'information dans laquelle nous glisserons les préoccupations liées à l'ergonomie et au graphisme'. À titre d'exemple, nous retenons les critères suivants :

- plan du site et agencement des contenus (garantissant un usage facile, et évitant les téléchargements longs)

- $\quad$ habillage du site (mise en page)

- $\quad$ lisibilité, typographie, etc.

- mise à jour, veille

- gestion des liens

Que dire de ces critères ? Pour le premier point de la liste, le Guide SAEL rappelle qu'une page d'accueil et un menu principal «ne doivent pas contenir trop d'unités d'information (sept est un minimum) pour ne pas surcharger la mémoire à court terme. Trois niveaux de sous-menus sont un maximum, si l'on souhaite éviter que l'utilisateur ne se perde » (Bonnet, 2009 : 38), or bien souvent, les sites FLE indépendants sont très chargés en information dès leur page d'accueil, quand ils ne sont pas parasités par les encarts publicitaires qui permettent de financer leur hébergement. L'habillage d'un site et les choix relatifs au graphisme sont des éléments déterminants pour sa réussite car ce sont eux qui favorisent en partie l'usage facile et la prise en main par les utilisateurs : l'internaute reviendra rarement sur un site qui aura été identifié comme ayant un « usage difficile». Pour être efficace, la veille doit permettre une mise à jour régulière des contenus et exploiter différents outils comme les flux $\mathrm{RSS}^{10}$, reconnus par l'icône@, les agrégateurs et tous types de réseaux sociaux susceptibles d'intéresser l'utilisateur. Enfin, concernant le référencement, rappelons l'importance des métadonnées, informations servant à décrire le contenu d'un site et qui seront ensuite utilisées par les moteurs de recherche pour le référencement du site. Plus la description du contenu sera pertinente, plus on augmentera les chances d'un site d'être mieux référencé et indexé. Il faut donc accorder aux méta-balises (ou méta-tags) toute leur importance en amont de la création proprement dite du site internet.

Quels sont les sites à destinations des apprenants et sont-ils nombreux dans le domaine du FLE ? Ils le sont, mais présentent une grande hétérogénéité. Les sites à destination des apprenants de français langue seconde/langue étrangère présentent des profils très variés en fonction des compétences visées, des objectifs et des attentes du public ciblé. Grâce à deux outils principalement, CITO et Quarkbase, on peut opérer un premier recencement, mais il ne s'agit là que d'une première étape à une recherche qui pourrait être conduite de façon plus systématique avec la mise en place d'une véritable veille. Voici à titre indicatif quelques uns des sites les plus connus, institutionnels ou pas, soit à destination des enseignants, soit à destination des apprenants, soit à destination des deux (avec une légère tendance à l'être malgré tout toujours plus pour les enseignants) :

\begin{tabular}{|l|l|}
\hline Nom du site & URL \\
\hline $\begin{array}{l}\text { Apprendre et enseigner la langue française (et } \\
\text { les autres sites de la « famille Perrot » comme } \\
\text { lexique FLE, Polar FLE ou Phonétique) }\end{array}$ & http://apprendre.over-blog.fr/ \\
\hline Apprendre le français en France & www.fle.fr \\
\hline Bonjour de France! Le magazine pour & www.bonjourdefrance.com \\
\hline
\end{tabular}




\begin{tabular}{|c|c|}
\hline pratiquer le français et dialoguer sur internet & \\
\hline EduFle.net, le site coopératif du FLE & http://www.edufle.net/ \\
\hline $\begin{array}{l}\text { FrançaisFacile.com, cours de français } 100 \% \\
\text { gratuits }\end{array}$ & $\underline{w w w}$.francaisfacile.com \\
\hline Le point du FLE, apprendre le français & http://www.lepointdufle.net/ \\
\hline RFI, langue française & http://www.rfi.fr/lffr/statiques/accueil_apprendre.asp \\
\hline TV5 Monde & $\begin{array}{l}\text { http://www.tv5.org/TV5Site/enseigner-apprendre- } \\
\text { francais/accueil apprendre.php }\end{array}$ \\
\hline $\begin{array}{l}\text { "Amélioration du français », Centre collégial } \\
\text { de développement de matériel didactique }\end{array}$ & http://www.ccdmd.qc.ca/fr/ \\
\hline
\end{tabular}

Tableau 1 : sites FLE et liens

Cette liste ne fait pas apparaître les cours de «Civilisation française de la Sorbonne » (Français Langue Etrangère) qui, malgré leur succès dû à la réputation internationale de l'Université Paris 1 , ne dévoilent pas leur contenu en ligne, la formation étant payante.

\section{Comparaison des techniques utilisées : quelques exemples de mises en oeuvre}

Dans le cadre d'une comparaison avec les sites internet d'ESL, il est nécessaire d'aborder la question des financements. Il est attesté que les moyens mis en œuvre par les Etats-Unis et la Grande Bretagne pour une politique linguistique de diffusion de l'anglais sont largement supérieurs à ceux des pays de la Francophonie, ce qui explique en partie les différences de prestations des sites internet. Dès la fin de la seconde guerre mondiale, la promotion de l'anglais comme langue véhiculaire internationale est devenue une priorité, si bien qu'organismes privés à but non lucratif (comme le Center for Applied linguistics) et organismes publics (comme le British Council) ont bénéficié de financements importants. Aussi, les sites que nous vous proposons d'étudier ne se situent peut-être pas dans la même catégorie que ceux précédemment cités, cependant, nous les trouvons riches d'enseignement :

- British Council : http://www.learnenglish.org.uk/

- BBC-Learning English : http://www.bbc.co.uk/worldservice/learningenglish/

- Cambridge English online : http://www.cambridgeenglishonline.com/

- Onestopenglish : http://www.onestopenglish.com/

(On notera que les cours de langue pour débutants de la BBC sont toujours les plus visités même s'ils exploitent la recette surannée du « Talk x language » proche de la méthode Assimil.)

Le premier site que nous vous invitons à explorer est celui de Onestopenglish. Il s'agit d'un site de ressources pédagogiques et interactives pour l'enseignement de l'anglais, dont les contenus sont pour la plupart accessibles gratuitement ou après inscription. Ce site très complet collabore en outre avec le journal The Guardian pour proposer des leçons thématiques liées à l'actualité, sur trois niveaux (élémentaire, intermédiaire et avancé).

Image 1 et $2:$ page d'accueil de Onestopenglish (http://www.onestopenglish.com/) et page collaborative avec The Guardian 


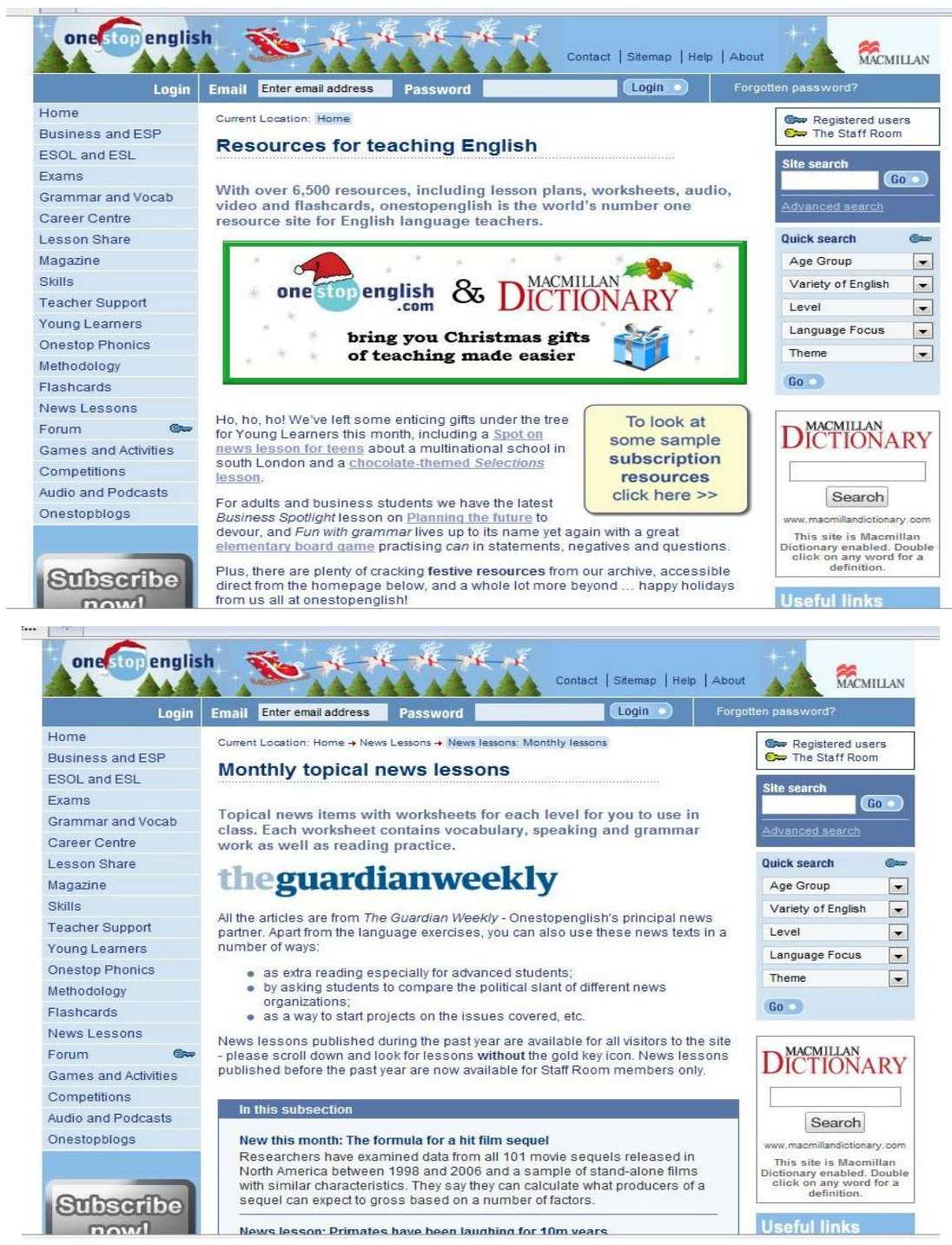

Il est intéressant de noter qu'il n'existe pas en France de tel partenariat avec les journaux Le Monde ou Libération par exemple. Le Monde a bien publié ces derniers mois quelques numéros hors série sur la langue française, mais son site internet privilégie la formation à l'anglais (voir onglet «Pratique »du ruban se situant en haut de la page d'accueil, puis sous-onglet «Formation anglais » qui vous conduit au GymGlish.com). En revanche, le site internet du journal The Guardian propose bien lui, sous l'onglet «Education », un sous-onglet «Tefl» soit Teaching English as a foreign language. Il nous semble que ces différences illustrent également des attitudes différentes vis-à-vis des publics migrants, et donc vis-àvis de la politique linguistique. Il y a là une sorte de paradoxe saisissant entre la volonté d'une Francophonie vivante et le manque de relais institutionnels (au sens large).

Le deuxième site que nous présentons est celui de la BBC, qui offre deux domaines distincts : « Teaching English» ( (http://www.bbc.co.uk/worldservice/learningenglish/). Nous prenons l'exemple de la tablette phonétique interactive qui a très largement attiré l'attention de nos futurs enseignants de FLE : ils y ont vu un outil essentiel pour leurs propres apprenants, mais ne connaissaient pas d'outil aussi performant pour le français (disponible sur internet) : 
Image 3 : Pronunciation $B B C$

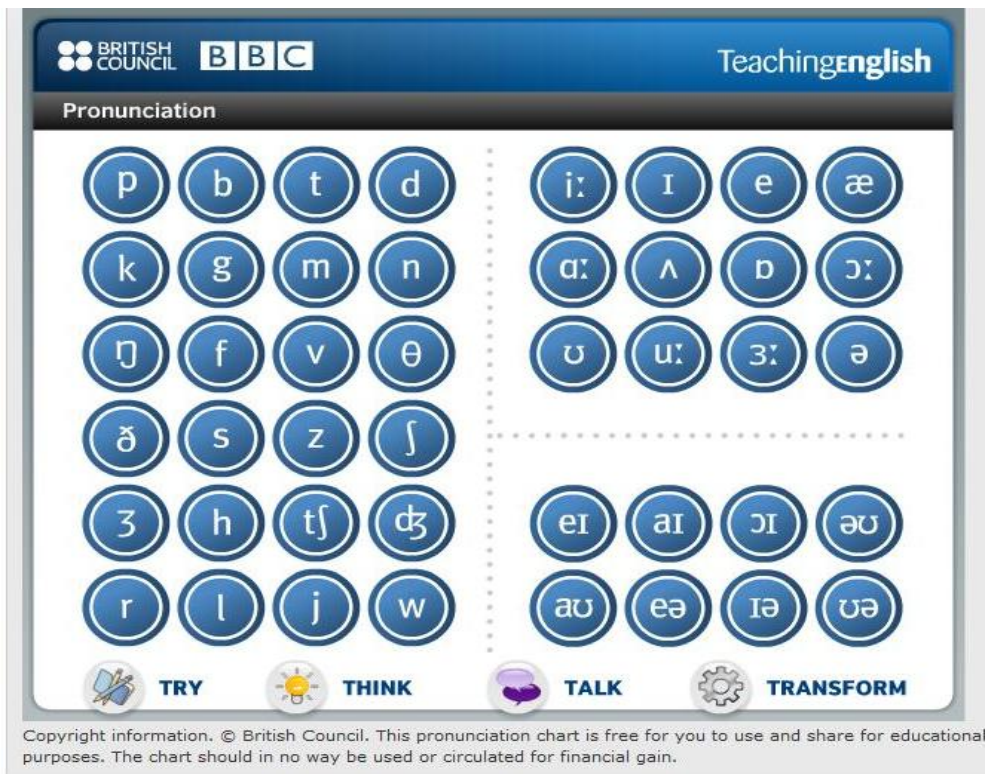

Les linguistes sauront bien sûr plus facilement repérer les défauts de ces supports (problèmes linguistiques, comme avec les sons de cette charte qui ajoutent des schwa aux réalisations des [p], [b], etc. ; lacunes ou laxisme, etc.), mais l'apprenant en retire la possibilité d'un entraînement intensif à domicile.

Notre troisième exemple s'appuie sur le site internet Cambridge English Online (http://www.cambridgeenglishonline.com/). L'image ci-dessous est celle de la rubrique «Phonetics Focus » qui comprend vingt activités ludiques pour travailler les sons de la langue anglaise :

Image 4 : Phonetics Focus (http://cambridgeenglishonline.com/Phonetics_Focus/)

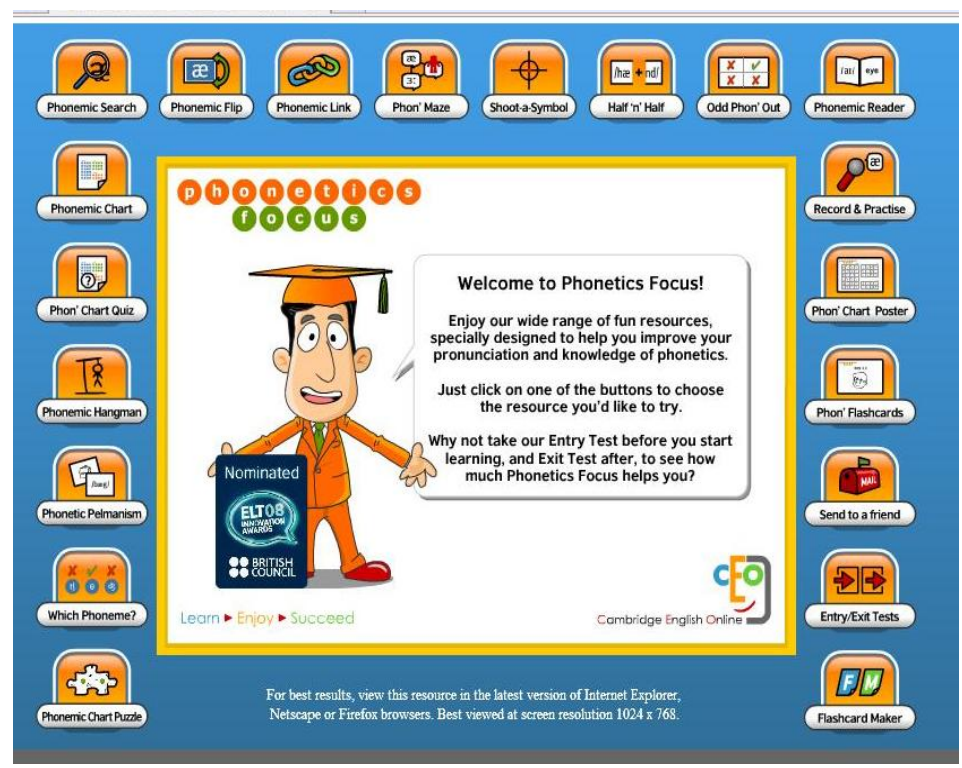

Le même site propose des jeux créatifs aussi performants que le Cartoon Maker. Certains étudiants de la formation FLE étaient cependant déçus de ne pouvoir l'utiliser avec leurs apprenants, le support n'acceptant pas les accents : 
Image 5 : Cartoon Maker (http://cambridgeenglishonline.com/Cartoon_Maker/)

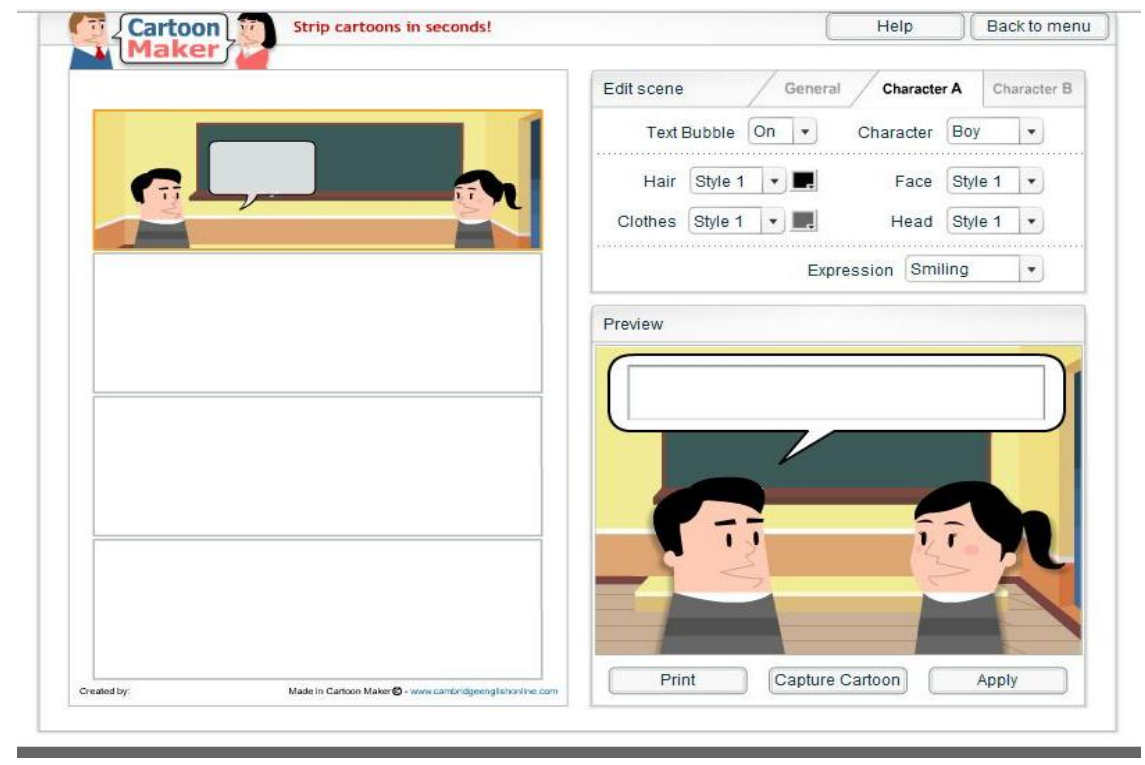

Pourquoi ces sites sont-ils si attractifs et si faciles d'utilisation? Nous avons identifié au moins un point commun entre ces trois exemples, que ne partagent pas la plupart des sites internet FLE (ce qui n'est pas le cas de la page "Amélioration du français » du Centre collégial de développement de matériel didactique (http://www.ccdmd.qc.ca/fr/ ). S'ils sont si attractifs aux yeux des utilisateurs, c'est parce qu'ils sont « vivants ", à l'image d'une langue vivante. Cette impression de dynamisme est rendue grâce à l'utilisation d'animations de type flash, les techniques flash étant excellentes pour ce type d'interfaces.

Nous ne rentrerons pas dans les débats sur les techniques du flash et les logiciels libres ou pas, mais remarquons simplement que ces techniques d'animation sont un moyen de rendre les sites FLE à destination des apprenants plus attractifs et plus faciles à utiliser. Nous n'établissons pas non plus d'adéquation aveugle entre cette observation et l'apprentissage linguistique proprement dit, mais soulignons bel et bien une corrélation entre politique linguistique visant à favoriser la diffusion de la langue française et efficacité/attractivité des sites internet. On peut néanmoins observer que se pose la question de l'impact de la technologie sur l'apprentissage : un site plus fonctionnel et dont le contenu sera plus facilement lisible n'aura-t-il pas plus de chance de conduire à une meilleure progression dans l'apprentissage de la langue française via l'utilisation de sites d'apprentissage en ligne?

\section{Propositions}

Le Ministère de l'Education Nationale (MEN), l'Organisation Internationale de la Francophonie (OIF) et la Fédération Internationale des Professeurs de Français (FIPF) ont été désignés par le Centre International d'Etudes Pédagogiques (CIEP) comme partenaires pour la création et l'animation de sites internet à destination des enseignants de langues. Ne pourrait-on pas imaginer l'Institut de Linguistique Française (ILF), la Délégation Générale à la Langue de France et aux Langues de France (DGLFL), le laboratoire Analyse et Traitement Informatique de la Langue Française (ATILF) et des universités francophones, comme des opérateurs/partenaires potentiels pour la création et l'animation de sites à destination des apprenants?

Par ailleurs, une proposition concrète doit s'accompagner d'une réflexion sur les coûts qui varieront en fonction de nombreux paramètres: «les coûts de développement correspondent essentiellement à la production des contenus et des éléments graphiques et multimédias. Les coûts de diffusion couvrent le déploiement de la plateforme, du LMS et des contenus. Les coûts de maintenance correspondent aux 
mises à jour des contenus et des logiciels» (Bonnet, 2009: 46), ce que le Guide SAEL propose de résumer sous la forme du schéma ci-dessous :

Tableau 2 : Les coûts à prendre en compte

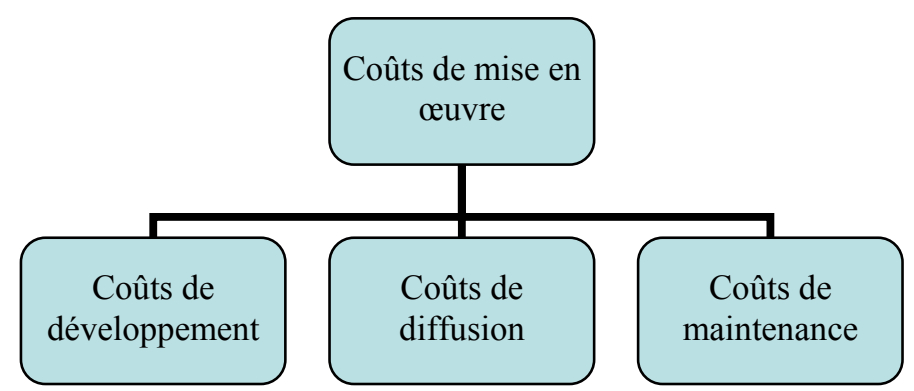

Comme le rappelle le Guide SAEL, la gestion d'un site est un métier qui nécessite des compétences spécifiques, et cette prestation a un coût conséquent pour toute la durée de vie d'un site. Ainsi, l'hébergement, les publications (i.e. le fait de pouvoir apporter des changements à un site) et la maintenance du $\mathrm{CMS}^{11}$ sont autant de paramètres à prendre en compte en plus du choix des partenaires et des créateurs (comme par exemple l'intervention de développeurs flash).

Des normes ne pouvant être établies dans le domaine de la création de sites individuels FLE, on pourrait en revanche proposer d'élargir les bourses ou les appels à création de site afin de financer des projets intéressants, ce qui permettrait en contre partie d'aider à la diffusion de certaines pratiques ou techniques dans la réalisation des sites internet d'apprentissage du français développés de façon indépendante.

\section{Conclusion}

\subsection{TICE, pratiques internet et évolution de la langue française}

Dans la lignée des travaux de J. Anis et J.-L. Lebrave sur les pratiques d'écriture en liens avec l'usage des nouvelles technologies (Lebrave, 2001 ; Anis et Lebrave, 1993), on s'interroge ici sur les transformations orthographiques du français (dans le cadre des nombreux débats toujours d'actualité sur les réformes de l'orthographe). Un sondage auprès des étudiants permet de vérifier certaines intuitions concernant les évolutions orthographiques de la langue entraînées ou appuyées par les nouvelles technologies. On savait déjà que le traitement de texte, en tant qu'outil incontournable pour le monde éducatif, ses praticiens et ses publics, allait entrainer certaines modificationss, et que cela est principalement lié à l'économie des logiciels, aux moyens mis en œuvre par les industriels pour élaborer les versions de leurs produits dans plusieurs langues, puis à la qualité des outils et des traductions, des dictionnaires installés, etc. Nous avons tous été confrontés dans notre pratique aux corrections orthographiques automatiques qui soulignaient intempestivement nos traits d'union, ou tendaient à vouloir faire disparaitre l'accent circonflexe de nos ' $i$ '. La question n'est alors pas vraiment celle d'être ou de ne pas être puriste : ce qui nous intéresse priroritairement, c'est la nécessité d'intégrer cette nouvelle donnée et ses conséquences dans un Observatoire des pratiques linguistiques.

Ainsi, nous souhaitons vérifier à l'avenir si l'usage rapide d'internet tend également à être un vecteur, un accélérateur des transformations orthographiques de la langue, plus qu'une réforme ne l'aurait espéré pour elle-même. Les étudiants, les chercheurs, les acteurs des métiers de l'information et de la communication (comme les journalistes), travaillent sur des documents de traitement de texte tout en étant la plupart du temps également connectés. Tout en rédigeant, ils peuvent à tout moment rechercher un document, une source, une référence, un lien, etc. sur la toile. Quand ils ont un doute sur l'orthographe d'un mot, ils vont parfois le taper dans un moteur de recherche et observent le résultat : le moteur fait une suggestion, qui respecte le principe de la plus grande occurrence, on est alors libre de choisir de conserver la suggestion (allant dans le sens de l'idée que la règle du plus grand nombre l'emporte). Cette règle, 
ironiquement, peut jouer des tours quand elle s'impose à la norme, à la forme de langue standard, voilà peut-être comment une forme, attestée mais non majoritaire initialement, a été élue par les internautes qui se sont mis à écrire «compte-rendu» avec un trait d'union quand pour une fois celui-ci n'était pas nécessaire.

\subsection{TICE et politique linguistique}

Ce processus vérifie que l'espace virtuel n'est pas une source écrite comme les autres : on la décrit le plus souvent comme une source non figée, non stable. Ceci confère un statut spécifique à son «écrit »: il s'agit d'un écrit mutable, dépendant des pratiques langagières de ses utlisateurs (plus encore que de l'oral). Une veille des suggestions lexicales sur internet par l'observatoire des pratiques linguistiques rendrait compte de ce que l'écrit est, ou peut être, lorsqu'il n'est pas uniquement un produit politique et idéologique. Sur internet se mélangent en effet toutes les formes d'écrit : des écrits légitimes aux écrits non standards, en passant par les langues mixtes, toutes les variétés et tous les phénomènes sont représentés, et l'on peut se demander à juste titre si ces écritures ne vont pas modifier les processus d'évolution de la langue.

Enfin, parmi les explications généralement invoquées pour expliquer les différences de compétence en langue chez les apprenants de français langue étrangère (fussent-ils individus non francophones en mobilité ou migrants en pays francophones), on trouve généralement les écoles fréquentées antérieurement ou le temps passé à parler le français en situation professionnelle, familiale ou amicale, auxquelles on peut aujourd'hui ajouter l'accès aux ressources internet. Or, plus les sites de FLE seront attractifs et efficaces, plus les futurs locuteurs auront la possiblité d'augmenter leur compétence linguistique en français. Pour saisir l'importance des sites internet à destination des apprenants, il est donc nécessaire d'envisager chaque internaute comme apprenant potentiel d'une langue. C'est ce qui se passe tous les jours quand un internaute se demande s'il serait capable de surfer sur le web dans telle ou telle langue. Ce petit voyage virtuel conduit bien souvent l'internaute à explorer des pages sans connaissance linguistique, la démarche initiale étant toujours grandement facilitée par les suggestions lexicales des moteurs de recherche. Ainsi, l'internaute tentera d'identifier dans un nouvel univers linguistique, les jalons de sa toile quotidienne, à travers des lieux et des empreintes partagées internationalement comme les réseaux sociaux, la presse, et les sites institutionnels. Cette méthode heuristique conduit plus souvent qu'on ne le croit à taper "apprendre » une langue dans sa propore langue ou en français (à plus forte raison si elle est la langue cible) ou en anglais, la langue véhiculaire ${ }^{12}$, et pourquoi pas «apprendre le français ». Quelque soit la langue utilisée pour rechercher un site d'apprentissage, les sites de FLE devraient alors pouvoir être présents et identifiés comme des sites attractifs sur lesquels on a envie de retourner pour apprendre la langue française.

\section{Références bibliographiques}

Anis, J. et J.-L. Lebrave (éds). (1993). Texte et ordinateur : les mutations du lire-écrire. Paris : CRL, Université de Paris X-Nanterre.

Bonnet, A. (coord. par). (2009). Guide SAEL. Paris : CIEP/ANIF.

Carpenter Binkley, S.et J. E. Hall. (2003). « Sound Pedagogical Practice on the Web», The French Review, 76, 3, 564-579.

CITO, moteur de recherche de ressources pédagogiques FLE (CUEF, Université Grenoble 3) : http://domus.grenet.fr/cuef/cito/citoprof/accueil.html

Educnet (enseigner avec le numérique), « Normes et standards » : http://tice.education.fr/services/normes-tice/

Gilbert, D. et alii. (2003). Guide de conception pédagogique et graphique d'un site éducatif sur le réseau Internet, version 4.1. APTIC, Université Laval [Disponible sur: http://www.aptic.ulaval.ca/guidew3educatif/, consulté le 28 décembre 2009]. 
Goullier, F. (2006). Les outils du Conseil de l'Europe en classe de langue : Cadre européen commun et Portfolios. Paris : Editions Didier.

Grandbastien, M. (2004). « Premiers pas dans le monde des standards pour la formation en ligne. Paradoxes, défis et propositions », Distances et savoirs, 4, 2, 395-408.

Lebrave, J.-L. (2001). « Comment écriront-ils ? », Diogène, 4, 196, 163-171

Louveau, E. et F. Mangenot. (2006). Internet et la classe de langue. Paris : CLE International.

Un cadre européen commun de référence pour les langues : apprendre, enseigner, évaluer. (2001). Paris : Conseil de l'Europe/Éditions Didier [Disponible sur: http://www.coe.int/T/DG4/Linguistic/Source/Framework_FR.pdf, consulté le 28 décembre 2009]

Ushida, E. (2005). «The Role of Students' Attitudes and Motivation in Second Language Learning in Online Language courses », CALICO Journal, 23 (1), 49-78.

Quarkbase : http://www.quarkbase.com/

${ }^{1}$ Cadre Européen Commun de Référence pour les Langues.

${ }^{2}$ « Le E-learning correspond à l'utilisation des technologies numériques pour améliorer la qualité de la formation en facilitant l'accès à des ressources et des services, des échanges et une collaboration à distance. C'est le recours aux technologies pour proposer des ressources, des services et des modules de formation via internet» (Bonnet, 2009 : 73-74).

${ }^{3}$ Par ailleurs, j'ai soumis un questionnaire à mes étudiants de Master 1 FLE de l'Université d'Angers à l'automne 2009 portant sur la formation des enseignants de FLE dont les résultats sont en cours de traitement.

${ }^{4}$ Nous nous référons ici aux niveaux communs de référence (de $\mathrm{A} 1 \mathrm{à} \mathrm{C} 2$ ) du Cadre européen commun de référence pour les langues (voir bibliographie).

${ }^{5}$ Voir la Charte ergonomique des sites Internet publics. Ministère du Budget, des Comptes publics et de la Fonction publique. [En ligne] http://www.references.modernisation.gouv.fr/sites/default/files/Charte ergonomique_v2.0_2.pdf, consulté le 28 décembre 2009.

${ }^{6}$ Les résultats $C I T O$ se trouvent ici : http://domus.grenet.fr/cuef/cito/citoprof/resultats.php, consulté pour la dernière fois le 28 décembre 2009.

${ }^{7}$ Recherches associées à « apprendre le français » proposées par Google : apprendre le français gratuitement; jeux pour apprendre le français; comment apprendre le français; logiciel pour apprendre le français; apprendre le français en ligne ; apprendre le français pour enfant ; learn french.

${ }^{8}$ Voir http://www.xtec.cat/ \%20sgirona/index.htm.

${ }^{9}$ « En informatique, l'ergonomie vise à ce que les informations affichées sur l'écran, via l'interface graphique, soient accessibles pour les utilisateurs » (Bonnet, $2009: 74)$.

${ }^{10}$ Le flux RSS permet la syndication de contenu web. «Le standard RSS représente un moyen simple d'être tenu informé des nouveaux contenus d'un site web, sans avoir à le consulter » (Bonnet, $2009: 75)$.

${ }^{11}$ Acronyme de Content Management System : «Le CMS est une application logicielle, destinée à la conception d'applications multimédias ou de sites web et à leur mise à jour dynamique. » (Bonnet, 2009 : 73).

${ }^{12} \mathrm{Ce}$ qui par ailleurs souligne l'importance des interfaces en plusieurs langues nécessitant des efforts de traduction. 